\title{
TAGUNG
}

\section{Quo vadis Europa? Europäische Integrations- und Migrationspolitik in Vergangenheit, Gegenwart und Zukunft}

\author{
Carsten Albers*
}

Am 6. und 7. Mai 2010 fand in Bremen mit Unterstützung des ,Zentrums für Europäische Rechtspolitik der Universität Bremen“, des „Bundesamtes für Migration und Flüchtlinge“ sowie des „Arbeitskreises Europäische Integration e.V.“ die Tagung „Wechselwirkungen von Migration und Integration im europäischen Mehrebenensystem" statt. Den Anstoß für die Veranstaltung - so der Tagungsinitiator Klaus Sieveking - gab das Lissabon-Urteil des Bundesverfassungsgerichts, das die Debatte um die Einflussnahme nationaler Parlamente auf Europa und den europäischen Integrationsprozess neu entfacht habe.

Im Mittelpunkt sollten die gestiegenen Anforderungen an Europa stehen, die aus der Globalisierung der Arbeitsmärkte folgen und durch die das internationale und nationale $\mathrm{Zu}$ wanderungsrecht geprägt werden. Hierzu wurden Fragen der Arbeitsmigration, der Freizügigkeit in der Europäischen Union, der Einbürgerung, der politischen Teilhabe und der Integration von Migranten diskutiert. Die unterschiedlichen Steuerungsdimensionen der Migrationspolitik sollten dabei fächerübergreifend aus rechtlicher und politisch-soziologischer Sicht betrachtet und damit ein interdisziplinärer Dialog geführt werden.

Zuwanderungskonzepte: ihre ökonomische, politische und rechtliche Bedeutung

Petra Bendel stellte in ihrem Referat die Widersprüche zwischen Migration und Integration im Mehrebenensystem der Europäischen Union heraus. Im Schwerpunkt diskutierte sie die europäische Asyl- und Flüchtlingspolitik

\section{Wechselwirkungen von Migration und Integration im europäischen Mehrebenensystem}

Bremen, 6./7. Mai 2010

\section{Begrüßung}

Prof. Dr. Felix HERZOG, Dekan Fachbereich Rechtswissenschaft, Direktor des Zentrums für Europäische Rechtspolitik, Universität Bremen Prof. Dr. Klaus SIEVEKING, Universität Bremen

Eröffnungsvortrag: Widersprüche zwischen Migration und Integration im Mehrebenensystem

PD Dr. Petra BENDEL, Universität ErlangenNürnberg

Zuwanderungskonzepte: ihre ökonomische, politische und rechtliche Bedeutung

Migration Hochqualifizierter

Dr. Hans Dietrich VON LOEFFELHOLZ, Bundesamt für Migration und Flüchtlinge, Nürnberg

\section{Zirkuläre Migration}

Dr. Frithjof ZERGER, Bundesministerium des Innern, Berlin

Free movement of workers

Prof. Dr. Elspeth GUILD, Radboud Universiteit Nijmegen

Moderation: Prof. Dr. Holger HOFFMANN, European Legal Network on Asylum (ELENA), Fachhochschule Bielefeld

Zuwanderung und ihre gesellschaftspolitische Bedeutung

Political participation

Prof. Dr. Kees GROENENDIJK, M.A., Centre for Migration Law, Radboud Universiteit Nijmegen

Einbürgerung

Prof. Dr. Dr. h.c. Kay HAILBRONNER, Universität Konstanz

Moderation: Dr. Christiane FALGE, Interkulturelles Gesundheitsnetzwerk (IGN) Bremen

\footnotetext{
* Carsten Albers, Wissenschaftlicher Mitarbeiter am Fachbereich Rechtswissenschaft der Universität Bremen.
} 
und kritisierte die unterschiedlichen Standards der Mitgliedstaaten beim Umgang mit Asylantragstellern und Flüchtlingen. Es gebe einheitliche EU-Richtlinien zum Umgang mit Asylsuchenden, die allerdings nicht überall gleich umgesetzt würden. Zwar habe sich die Lage der Asylsuchenden und der Flüchtlinge in Europa seit dem Inkrafttreten des Vertrages von Lissabon grundsätzlich verbessert, die Mitgliedstaaten versuchten jedoch weiterhin, nationale Interessen durchzusetzen. Es herrsche eine „Asyllotterie“ vor, weil nicht überall nach gleichartigen Standards Asyl gewährt werde. Insoweit sei zu fordern, dass die Mitgliedstaaten in der Beantwortung dieser Fragen „endlich mit einer Stimme“ sprächen. Eine einheitliche Asylpolitik sei immer noch nicht ersichtlich, was das Mehrebenensystem der Europäischen Union weiterhin zu einem spannenden Forschungsfeld mache - gerade seit den neuen Bedingungen durch den Vertrag von Lissabon.

Hans Dietrich von Loeffelholz, problematisierte Konzepte und Praxis der Zuwanderung Hochqualifizierter nach Deutschland. Er stellte hierbei auf die bestehenden Interdependenzen im europäischen Mehrebenensystem ab. Migration werde verstärkt durch Mobilität ersetzt: Hochqualifizierte seien heute sehr mobil und blieben lediglich einige wenige Jahre im Land. Hieraus entwickelte er die Fragestellung, ob unter derartigen Bedingungen überhaupt ein Integrationsbedarf für den genannten Personenkreis besteht. AnschlieBend stellte er heraus, dass die Steuerung der Zuwanderung von Hochqualifizierten im Mehrebenensystem untereinander Abstimmung, Konsistenz der rechtlichen Rahmenbedingungen und nachhaltige politische Konzepte erfordere. Es sei auf deutscher Ebene zu konstatieren, dass die „Qualität der Arbeitsmigration" durch die zur Steuerung eingesetzten Instrumente zugenommen habe. Es müsse in Deutschland und der Europäischen Union jedoch eine stärkere „Willkommens-, Integrations- und Anerkennungskultur" entwickelt werden.
Eröffnungsvortrag: Begriff der Integration Konflikte und Tragweite

Prof. Dr. Dietrich THRÄNHARDT, Universität Münster

Moderation: Libuse CERNA, Bremer Rat für Integration

Europäische Migrations- und Integrationsstrukturen im Spannungsfeld globaler Migration

Christiane WESTPHAL, Referentin, Generaldirektion Beschäftigung der Europäischen Kommission, Brüssel

Moderation: Jenni WINTERHAGEN, Bremen International Graduate School of Social Sciences (BIGSSS), Universität Bremen

Concrete aspects in selected member states Niederlande

Dr. Tineke STRIK, Centre for Migration Law, Radboud Universiteit Nijmegen

Frankreich

Dr. Yves PASCOUAU, Université de Pau et des Pays de l'Adour

Moderation: Jenni WINTERHAGEN, Bremen International Graduate School of Social Sciences (BIGSSS), Universität Bremen

Die Bedeutung der Menschenrechte am Beispiel des Schulbesuchs von nicht-dokumentierten Kindern und Jugendlichen in Deutschland

Dr. Felix HANSCHMANN, Max-Planck-Institut für ausländisches öffentliches Recht und Völkerrecht, Heidelberg, zurzeit Bundesverfassungsgericht, Karlsruhe

Moderation: Prof. Dr. Klaus SIEVEKING, Universität Bremen

Frithjof Zerger setzte den Schwerpunkt seines Vortrags bei der Verbindung von Migrationsund Entwicklungspolitik. Hierbei stellte er auf das derzeit unter Nichtregierungsorganisationen und Menschenrechtsaktivisten intensiv diskutierte Konzept der zirkulären Migrationspolitik ab. Dieser Begriff umfasse wiederholte Wanderungsbewegungen zwischen dem Heimatstaat und dem Staat der Erwerbstätigkeit. Es gehe um fortgesetzte temporäre Aufenthalte in zwei oder mehreren Staaten. Zirkuläre Migration sei insoweit vom herkömmlichen Gastarbeiterverständnis abzugrenzen. Die Mitgliedstaaten der Gemeinschaft seien sich allerdings nicht einig, wie man mit der Idee der zir- 
kulären Migration umgehen solle. Zerger hob den möglichen Nutzen, aber auch denkbare Risiken des Konzepts hervor. Bei dessen Umsetzung gebe es offene Fragen, zum Beispiel wie lange der Aufenthalt im Zielstaat dauern solle, an welche Zielgruppen sich das Konzept wende, wie die Rückkehr in den Heimatstaat gesichert werden könne und welche menschenrechtlichen Implikationen damit verbunden seien. Als problematisch erweise sich die Tatsache, dass gut ausgebildete Fachkräfte aus Entwicklungsstaaten in die Europäische Union abwanderten und daher am Aufbau ihrer Heimatländer nicht mitwirken könnten. Gleichwohl biete das Konzept eine Reihe von Chancen.

Elspeth Guild zentrierte ihren Beitrag auf den ökonomischen Kern von Migration im europäischen Kontext und widmete sich in diesem Zusammenhang der gemeinschaftsrechtlichen Arbeitnehmerfreizügigkeit. Sie stellte hierzu die mit den 1957 unterzeichneten Verträgen von Rom eingeleitete Entwicklung des Rechts für Unionsbürger, innerhalb der Europäischen Union zu arbeiten, bis zur Gegenwart dar und hob die damit einhergehende Erweiterung der Statusrechte ehemaliger Drittstaatsangehöriger hervor. Als Beleg hierfür zeichnete sie unter anderem den Inhalt der Verordnungen über die Freizügigkeit der Arbeitnehmer innerhalb der Gemeinschaft von 1964 und 1968 sowie der Richtlinie zur Aufhebung der Reise- und Aufenthaltsbeschränkungen für Arbeitnehmer der Mitgliedstaaten und ihrer Familienangehörigen und deren Anwendung in der Praxis nach. Ihrer Ansicht nach waren vor allem der Inhalt des Arbeitnehmerbegriffs und seine Auslegung durch den EuGH entscheidend. Im Fokus ihrer Überlegungen stand die Frage, welchen Effekt die stete Erweiterung der Gemeinschaft auf die Arbeitnehmerfreizügigkeit als Teilaspekt des „fundamentalsten europäischen Projekts" - des Binnenmarkts - hatte, hat und haben wird.

\section{Zuwanderung und ihre gesellschaftspolitische} Bedeutung

Kees Groenendijk sprach politische Partizipationsmöglichkeiten der Zuwanderer als Spiegel der Integrations- und Gesellschaftspolitik an. Er stellte dabei auf das Wahlrecht als Schlüssel zur gesellschaftlich-politischen Integration ab. Die derzeitige Situation in Europa sei gespalten. Nach Auffassung der einer politischen Partizipation für Zuwanderer gegenüber eher restriktiv eingestellten Länder ${ }^{1}$ führe das Gewähren eines Wahlrechts zu einer Verminderung des Interesses bei Zuwanderern, die Staatsangehörigkeit des Aufenthaltsstaates anzunehmen. Empirisch habe sich jedoch gezeigt, so Groenendijk, dass das Interesse an der Annahme der Staatsangehörigkeit des Aufenthaltsstaates nicht negativ von einem schon vorher bestehenden Wahlrecht beeinflusst werde. Ganz im Gegenteil sei in vielen Staaten, die ein Wahlrecht einräumten, die Zahl der Einbürgerungen nach der Gewährung des Wahlrechts stark gestiegen. Daher sei ein entscheidendes Argument für die Einräumung von Wahlrechten, dass sie als „Pfad“ zu begreifen seien, der zur Einbürgerung hinführe und nicht von ihr weg. Ein anderes Problem bestehe in Staaten, in denen Nicht-Unionsbürger kein Wahlrecht hätten und dem Erwerb der Staatsangehörigkeit Einbürgerungstests, vorgeschaltetet' seien. Da regelmäßig lediglich die hochqualifizierten, gut ausgebildeten Migranten diese Tests bestünden, hätten sie einen leichteren Zugang zu politischer Partizipation. Ob dieser Effekt so gewollt sei, sei fraglich.

Kay Hailbronner befasste sich mit der Bedeutung der Einbürgerung für den Integrationsprozess von Migranten. Politisch sei schon immer umstritten gewesen, ob Integration zur Voraussetzung für einen Einbürgerungsanspruch gemacht werden könne - klar sei aber, dass die Verleihung der deutschen Staatsangehörigkeit die Integrationschancen in der

1 Hier wurden Bulgarien, Österreich, Frankreich, Georgien, Griechenland, Italien, Lettland, Malta, Polen, Rumänien, die Tschechische Republik und Zypern genannt. 
zweiten und dritten Zuwanderergeneration unterstütze. Ob die Reform des deutschen Staatsangehörigkeitsrechts insoweit als Erfolg zu werten sei, lasse sich nicht sagen. Allerdings sei im Zeitraum von 2000 bis 2008 ein Rückgang der Einbürgerungszahlen von 186.000 auf nun 94.000 zu verzeichnen gewesen. Monokausale Erklärungsversuche hierfür griffen aber immer zu kurz. Weiter ging Hailbronner auf das „Problem der Mehrstaatigkeit" ein, welches er anhand des Optionsmodells des $\S 4$ Abs. 3 Nr. 1 und 2 in Verbindung mit $§ 29$ Staatsangehörigkeitsgesetz skizzierte. Interessant werde es in Zukunft, wenn Fälle der Mehrstaatigkeit vor die Verwaltungsgerichte kämen. Diese werden dann - so Hailbronner - im einstweiligen Rechtsschutz zu entscheiden haben, dass die deutsche Staatsangehörigkeit vorläufig bestehen bleibt. 52 Prozent aller Einbürgerungen würden heute unter Hinnahme der Mehrstaatigkeit durchgeführt - also stelle die Mehrstaatigkeit nicht die Ausnahme, sondern die Regel dar. Die insbesondere mit der Mehrstaatigkeit verbundenen rechtlichen Schwierigkeiten seien dabei - abgesehen von gelegentlich auftretenden Schwierigkeiten bei der Anwendung völkerrechtlicher Verträge - alle lösbar.

\section{Begriff der Integration - Konflikte und Trag- weite}

Zum Begriff der ,Integration“ referierte zu Beginn des zweiten Tagungstages Dietrich Thränhardt, der seinen Vortrag mit der Frage nach der Sinnhaftigkeit von Integration einleitete. Hierbei verwies er auf amerikanische Migrationserfahrungen und kam zu dem Schluss, dass Integration - oftmals leider nur als Anpassung verstanden - nicht immer und in jeder Hinsicht vorteilhaft sein müsse. Dies gelte gerade in den Konstellationen, in welchen sich die Aufnahmegesellschaft „,negativ“ auf Zuwanderer auswirke: Familienbindungen würden in anderen Gesellschaften größer geschrieben als in den westlichen Einwanderungsnationen. Auch werde das Alter mehr geachtet, sodass eine Integration in eine die genannten Punkte vernachlässigende Gesell- schaftsstruktur nachteilig für Zuwanderer sein könne. In diesem Zusammenhang sprach Thränhardt von „Akkulturationsproblemen“: Wenn Migranten ihre Kultur ,mitbringen " was ein aus der Migrationsgeschichte bekanntes Phänomen sei - werde dies häufig negativ gewertet. Integration sei mehr als Anpassung an die westliche Gesellschaft. Es dürfe diese „Defizitperspektive“, aus der alles, was Migranten mitbrächten, negativ aufgefasst werde, nicht geben. Der Integrationsdiskurs laufe sonst auf Assimilation hinaus, weil Integration stets mit der Vorstellung einer homogenen Gesellschaft assoziiert werde. Diese Idee sei unrealistisch: Einwanderer seien ,anders', und die Gesellschaft würde ärmer, wenn Einwanderer lediglich, angepasst" seien. Daher sei es auch unsinnig zu fordern, Einwanderer müssten in allen Berufsgruppen proportional vertreten sein. Eine Differenzierung werde dort zum Problem, wo sie sich zu Ausgrenzung verforme. Auch die politische Integration wurde von Thränhardt thematisiert: Es gebe sie bei Zuwanderern zwar praktisch nicht, aber dennoch seien Parteipräferenzen erkennbar. Als positives Beispiel für mehr politische Integration von Migranten sei das Betriebsverfassungsrecht anzuführen: Seitdem es dort das volle Wahlrecht gebe, sei die Zahl der Betriebsratsvorsitzenden mit Migrationshintergrund stetig gestiegen. Dies spreche für die Eröffnung von Partizipationsmöglichkeiten auf weiteren politischen und gesellschaftlichen Ebenen. Abschließend stellte Thränhardt fest, dass die Struktur der Gesellschaft für Integration wesentlich bedeutsamer sei als einzelne Integrationsmaßnahmen. Das Ausbildungssystem und das soziale Sicherungssystem etwa müssten den Gedanken der Integration verinnerlichen und widerspiegeln. Integration sei ein Prozess, der nie ,abgeschlossen" werde.

Europäische Migrations- und Integrationsstrukturen im Spannungsfeld globaler Migration

Christiane Westphal referierte über europäische Migrations- und Integrationsstrukturen 
im Spannungsfeld globaler Migration. Nach Westphal liegt die Nettoeinwanderung in die Europäische Union bei etwa 1,5 Millionen Personen pro Jahr. Die Gemeinschaft benötige Zuwanderung - es fehlten in den nächsten zahn Jahren circa 14 Millionen Arbeitskräfte, würde es keine Zuwanderung geben. Der europäische Integrationsprozess sei aber immer im globalen Kontext zu verstehen. Die Mobilität von Kapital und Arbeit steige stetig, was zu Verschiebungen in der globalen Arbeitsteilung führe. Die Motive für Einwanderung differierten dabei: Während Migration innerhalb der Europäischen Union stark geprägt sei vom Gedanken der Arbeitsanreize (,,wo es Arbeit gibt, da gehen die Ströme hin"), sei dies bei Drittstaatsangehörigen zum Teil anders. Bei nach Frankreich eingewanderten Drittstaatsangehörigen etwa komme nur jeder fünfte um Arbeit zu suchen. Familienzusammenführung sei ein ebenso großes Thema. Der Arbeitsmarkt sei für Drittstaatsangehörige regelmäßig nicht die „Stimulanz", um einzuwandern. Wie wichtig Zuwanderung für die innereuropäische Wirtschaft ist, verdeutliche die Tatsache, dass Einwanderer verantwortlich seien für etwa ein fünftel des Wirtschaftswachstums in der Europäischen Union. Sie seien primär im Gastronomie- oder Baugewerbe beschäftigt. Diese Zweige seien von der Krise in hohem $\mathrm{Maß}$ betroffen, wodurch Migranten die Folgen der Krise besonders häufig zu spüren hätten. Das träfe vor allem niedrig qualifizierte junge Männer. Vor diesem Hintergrund sei Einwanderung in die Europäische Union zwar grundsätzlich positiv. Man dürfe aber die $\mathrm{Au}-$ gen nicht vor den damit verbundenen Problemen verschließen: Armut und soziale Ausgrenzung seien verbreitet; hochqualifizierte Migranten übten regelmäßig eine Tätigkeit aus, die unter ihrer beruflichen Qualifikation liege, was auch als eine Folge der fehlenden Anerkennung von ausländischen Abschlüssen anzusehen sei. Strukturelle Probleme kämen hinzu: So sei eine sich längerfristig verfestigende Folge von Migration, dass die Integration der sogenannten zweiten Generation der Zugewanderten pro- blematisch sei. Bei dieser Personengruppe, deren (niedrig qualifizierten) Eltern beide keine Unionsbürger seien, liege die Beschäftigungsquote bei unter 50 Prozent (bei den Frauen liege sie sogar unter 40 Prozent). Experten gingen zudem davon aus, dass bereits in 10 Jahren das Angebot an Arbeitsplätzen in der Europäischen Union für die nur eine geringe Qualifikation benötigt werde um 85 bis 90 Prozent geschrumpft sein werde. Das bedeute, dass die Chancen für diese Personengruppe weiter sinke, weshalb die Mitgliedstaaten mehr investieren müssten, um bessere Bildung zu gewährleisten.

\section{Mitgliedstaatliche Konkretisierungen}

Bei dem folgenden Panel standen konkrete migrations- und integrationsspezifische Themen aus den Niederlanden und Frankreich im Mittelpunkt. Die Referenten behandelten schwerpunktmäßig die Bedeutung von Integrations- und Einbürgerungstests und bezogen sich hierbei auf Forschungsergebnisse, die sie in dem gemeinsam durchgeführten länderübergreifenden vergleichenden Forschungsprojekt INTEC (,Integration and Naturalisation Tests, the New Way to European Citizenship") der Radboud Universiteit Nijmegen erarbeitet haben.

Tineke Strik erläuterte die niederländischen Erfahrungen mit dem 2003 eingeführten System der ,researchtests'. Stadiumsabhängig hielten die Niederlande drei solcher Tests bereit: Bei erstmaliger Einreise, bei Aufenthaltsverlängerung und für einen Einbürgerungsantrag sei jeweils das Bestehen eines Tests erforderlich. Von 1993 bis 2000 sei debattiert worden, ob das damalige Einbürgerungssystem die Einbürgerung, zu leicht' mache. Im Jahre 2003 sei daher ein Einbürgerungstest eingeführt worden, der die Fähigkeit, Niederländisch zu sprechen und zu schreiben, abfrage sowie die ,social orientation“ des Bewerbers überprüfe. Bereits 1998 hätten die Niederlande Erfahrungen mit derartigen Tests gesammelt. Im Rahmen des „Newcomers Integration Act" (NIA) wurden seinerzeit Neu- 
ankömmlinge einem nicht verpflichtenden Integrationstest unterzogen, den nur wenige von ihnen bestanden. Dies habe an strukturellen Problemen gelegen, die mit solchen Kursprogrammen oftmals verbunden seien. So seien die Kurse nicht ,maßgeschneidert" gewesen und passten daher nicht auf jeden Migranten in gleicher Weise. Ferner seien der Kursteilnahme oft lange Wartezeiten vorgelagert gewesen. Diese Probleme habe die Politik aber nicht erkannt. Sie habe aus den hohen Durchfallquoten vielmehr geschlossen, dass die Tests deswegen schlecht ausfielen, weil keine Besuchspflicht der vorbereitenden Kurse bestehe. 2003 sei dann ein neues System eingeführt worden. Dies sei durch den „Integration Act" 2007 nochmals überarbeitet worden. Nun bestehe für alle Migranten zwischen 18 und 65 Jahren, die sich langfristig in den Niederlanden aufhalten möchten, eine Pflicht, den Test zu bestehen. Allerdings seien Ausnahmen vorgesehen. Seit dem 1. Januar 2010 müssten alle Migranten (,newcomers' und ,oldcomers') den Test innerhalb von dreieinhalb Jahren machen. Entziehe man sich dieser Pflicht, habe dies negative Konsequenzen und es drohten Strafgelder in Höhe von 250 bis 1.000 Euro, welche auch im Falle eines späteren Bestehens des Tests nicht zurückgezahlt würden. Die Auswirkungen der Verpflichtung zum Besuch der Kurse und zum Bestehen des Tests würden unterschiedlich bewertet: Zwar bestünden 70 Prozent der Hochqualifizierten und 60 Prozent der Niedrigqualifizierten den Test, was die Regierung dazu veranlasse, die Einführung des flächendeckenden Tests als Erfolg zu betrachten. Andererseits zögen die Tests einen Effekt nach sich, den Strik als „,nachteiligen Wechsel“ bezeichnete: Die Zahlen offenbarten, dass reiche, hochqualifizierte und junge Menschen den Test leicht(-er) bestehen. Die „übrigen“ blieben auf „der Strecke“. Daher seien die Auswirkungen von Einbürgerungstests stets aus mehreren Blickwinkeln zu betrachten.

Die französischen Erkenntnisse im Zusammenhang mit (Einbürgerungs-)Tests wurden von Yves Pascouau vorgestellt. Er erläuterte zunächst die Entwicklung der französischen Integrationspolitik in den vergangenen Jahrzehnten. 2006 sei ein neues Einwanderungsgesetz in Kraft getreten, welches eine weitreichende Reform der Einwanderungs- und Integrationspolitik dargestellt habe. Es habe unter anderem einen verpflichtenden Aufnahme- und Integrationsvertrag („Contrat d'accueil et d'intégration“, CAI) für Ausländer, die dauerhaft im Land bleiben wollen, vorgesehen. Dieser Integrationsvertrag sehe insbesondere die Teilnahme an Schulungen zu Grundwerten der Gesellschaft sowie an Sprachkursen vor. Der zur Teilnahme verpflichtete Adressatenkreis sei 2007 noch erweitert worden. Pascouau ging in seinem Vortrag sodann auf Migration zum Zwecke der Familienzusammenführung ein. Unter diesem Gesichtspunkt stellte er heraus, dass das geltende französische Recht inkompatibel mit diesbezüglichen gemeinschaftsrechtlichen Vorgaben sein könnte. Noch 2010 sei eine weitere Verschärfung des französischen Rechts zu erwarten, welche unter anderem eine weitreichende Verbreiterung der administrativen Kontrollmöglichkeiten mit sich bringe. Überdies stehe zu befürchten, dass die Anforderungen im Rahmen der Integrationsverträge weiter erhöht würden. Auch seien Änderungen des französischen Rechts im Hinblick auf die Verleihung und den Entzug der Staatsbürgerschaft geplant. Derzeit deute damit vieles in Richtung einer restriktiven $\mathrm{Zu}$ wanderungspolitik.

\section{Zur Bedeutung der Menschenrechte}

Im Schlussvortrag von Felix Hanschmann sollte die Bedeutung der Menschenrechte am Beispiel des Schulbesuchs von Kindern und Jugendlichen ohne Aufenthaltspapiere verdeutlicht werden. Am Beispiel dieses Themenkomplexes ließen sich - so Hanschmann - alle im Rahmen der Tagung betrachteten Normebenen zusammenfassend, wie in einem Brennglas “ zentriert betrachten. Er erläuterte, dass sich derzeit mehrere zehntausend Kinder ohne rechtmäßigen Aufenthaltsstatus in der Bundesrepublik aufhielten. Völker- 
recht, Bundesrecht, Landesrecht und interne Verwaltungsvorschriften wirkten auf dieses Sachgebiet ein. Eine Schulpflicht für nichtdokumentierte Kinder bestehe lediglich in Bayern und Nordrhein-Westfalen. Zentrales Problem sei dabei die nach herrschender Meinung wenigstens für die Schulleitung bestehende Verpflichtung nach $\S 87$ Abs. 2 Nr. 1 Aufenthaltsgesetz, nicht-dokumentierte Kinder der Ausländerbehörde zu melden. Danach haben öffentliche Stellen ,unverzüglich die zuständige Ausländerbehörde zu unterrichten, wenn sie im Zusammenhang mit der Erfüllung ihrer Aufgaben Kenntnis erlangen von dem Aufenthalt eines Ausländers, der keinen erforderlichen Aufenthaltstitel besitzt und dessen Abschiebung nicht ausgesetzt ist". Diese Meldeverpflichtung von Schulen führe dazu, dass nicht-dokumentierte Eltern ihre im schulpflichtigen Alter befindlichen Kinder nicht zur Schule schickten und den Kindern damit das Recht auf Bildung gänzlich genommen werde. Als Begründung für die Meldepflicht der Schulleitung und für einen Ausschluss nicht-dokumentierter Kinder aus dem Schulbetrieb werde - so Hanschmann - angeführt, der ,irreguläre Aufenthalt" der Kinder stehe der Gewährung staatlicher Leistungen entgegen. Weiter wird von den Befürwortern einer Meldepflicht angeführt, es müssten Widersprüche innerhalb der Rechtsordnung vermieden werden. Ferner stünden der Inanspruchnahme der staatlichen Leistung ,Schule' keine steuerliche Gegenleistung der nicht-dokumentierten Kinder beziehungsweise von deren Eltern gegenüber. Der Ausschluss der Kinder werde auch damit begründet, dass „keine Notwendigkeit“ der Beschulung bestehe, da der Aufenthalt ohnehin prekär und meist von nur kurzer Dauer sei. Unter diesen Voraussetzungen könnten „die Schulen ihren Bildungsauftrag nicht erfüllen“.

Diese ,Argumente' sind nach Auffassung des Referenten allesamt nicht tragfähig. Insbesondere die Tatsache, dass Kinder schon ihrem Wesen nach nicht für ihren ,illegalen' Aufenthalt in der Bundesrepublik verantwortlich zu machen seien, spreche evident dagegen, ihnen das Menschenrecht auf Bildung zu nehmen. Darüber hinaus könne verfassungs- und völkerrechtlicher Schutz nicht an den Aufenthaltsstatus geknüpft werden. Neben den offenkundigen verfassungsrechtlichen Verpflichtungen bestehe ein völkerrechtlicher Schutz des Rechts auf Bildung in vielfältiger Weise: Nach Art. 28 Abs. 1 der Kinderrechtskonvention der Vereinten Nationen, Art. 13 Abs. 1 und 2 in Verbindung mit Art. 2 Abs. 2 des Internationalen Paktes über wirtschaftliche, soziale und kulturelle Rechte, Art. 3 des Übereinkommens gegen die Diskriminierung im Unterrichtswesen sowie Art. 2 S. 1 des 1. Zusatzprotokolls zur Europäischen Menschenrechtskonvention sei die Bundesrepublik verpflichtet, auch nicht-dokumentierten Kindern Zugang zu Bildung zu gewähren. Aufgrund der Meldeverpflichtung der Schulleitung über den $\S 87$ Abs. 2 Nr. 1 Aufenthaltsgesetz und dem daraus folgenden Ausbleiben des Schulbesuchs einer Vielzahl von nicht-dokumentierten Kindern verstoße die Bundesrepublik im Ergebnis gegen verfassungs- und völkerrechtliche Verpflichtungen. Nicht-dokumentierte Kinder und Jugendliche müssten also in die allgemeine Schulpflicht einbezogen werden. $\S 87$ Abs. 2 Nr. 1 Aufenthaltsgesetz müsse geändert oder völkerrechtskonform ausgelegt werden: Weder Lehrkräfte noch die Schulleitung sollten verpflichtet sein, den irregulären Aufenthaltsstatus an Ausländer- und Polizeibehörden zu melden. Schließlich dürften Zwangsmaßnahmen, die bei Verletzungen der Schulpflicht ergriffen würden, nicht zur Aufdeckung des irregulären Aufenthalts führen.

Fazit - Erforderlichkeit Kritischer Migrations- und Integrationsforschung als Begleitinstrument der Europäischen Idee

In der von Klaus Sieveking geleiteten Abschlussdiskussion wurde herausgestellt, dass sich das fachübergreifende Konzept der Tagung gerade vor dem Hintergrund des Mehrebenensystems der Europäischen Union als richtig und wichtig erwiesen hat. Migration 
und Integration als „zwei Seiten derselben Medaille“ würden zentrale Themen der europäischen Gemeinschaft bleiben - daher sei deren Verrechtlichung auch weiterhin von Wissenschaftlern unterschiedlicher Fachgebiete kritisch zu begleiten. Nur wenn - auch unbequeme - Fragen fortwährend gestellt würden, könne dies letztlich einen auf allen Ebenen gleichermaßen erfolgreichen Integra- tionsprozess als Teil der Europäischen Idee herbeiführen. Auch in Zukunft sei daher eine fächerübergreifende Migrations- und Integrationsforschung von hervorragender Bedeutung für die Weiterführung der kontroversen Debatte um die Gestaltung der nationalen und europäischen Migrations- und Integrationsprozesse.

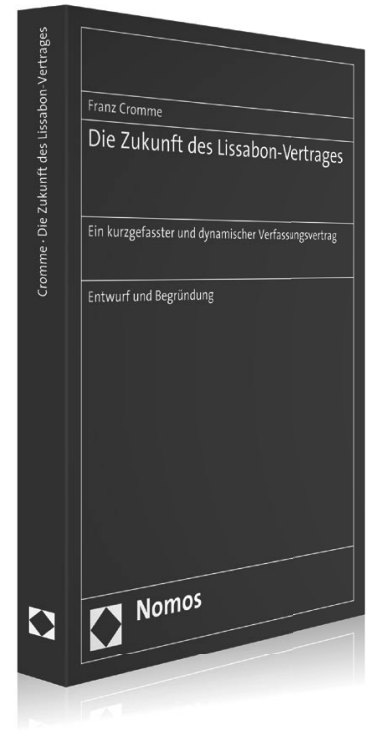

\section{Die Zukunft des Lissabon-Vertrages}

Ein kurzgefasster und dynamischer Verfassungsvertrag

Entwurf und Begründung

Von Dr. Franz Cromme, StSekr a.D.

2010, 325 S., brosch., 59,- $€$

ISBN 978-3-8329-5282-2

Das Buch mit der Fortführung des Lissaboner Vertrages in einem kurzgefassten Verfassungsvertrag ist zunächst - zusammen mit der umfassenden Begründung - vor allem ein kritischer Kommentar und ein schlankes Spiegelbild zum neuen Doppelvertrag der EU. Das Werk soll zugleich für die Zukunft einen konkreten Beitrag zur Theorie des Staatenverbundes leisten und wertet hierzu auch das Urteil des Bundesverfassungsgerichts vom Juni 2009 aus.

"Die Diskussion um Konzeption und Finalität der Europäischen Union wird weitergehen. Es ist zu wünschen, dass Vision und Gedanken Franz Crommes dazu einen Beitrag leisten werden."

RA Prof. Dr. Hans-Jürgen Rabe, NJW 41/10

Bitte bestellen Sie im Buchhandel oder versandkostenfrei unter $\boldsymbol{\text { www.nomos-shop.de }}$

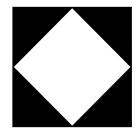

Nomos 